\title{
A Survey of Adolescent Mental Health from the Perspective of Sinology
}

\author{
Yifu Peng \\ No. 21 Middle School of Changsha, Changsha, China \\ Email: pengyf11@163.com
}

How to cite this paper: Peng, Y.F. (2019)

A Survey of Adolescent Mental Health from the Perspective of Sinology. Health, 11, 406-414.

https://doi.org/10.4236/health.2019.114036

Received: April 4, 2019

Accepted: April 25, 2019

Published: April 28, 2019

Copyright $\odot 2019$ by author(s) and Scientific Research Publishing Inc. This work is licensed under the Creative Commons Attribution International License (CC BY 4.0).

http://creativecommons.org/licenses/by/4.0/

\section{c) (i) Open Access}

\begin{abstract}
The purpose of this paper is to investigate the mental health status of adolescents. In this paper, the MHT test paper was used to evaluate the mental health status of 226 students in different places. MHT refers to the mental health test for primary and middle school students. Results: 1) 34 students had serious psychological tendency, which was mainly manifested in learning anxiety, anxiety about people, self-accusation tendency and impulse tendency; 2) There are gender differences in mental health problems among school students, with more girls than boys. There are also regional differences, with more students in Guangdong than in other regions. There are also differences in learning stages, and there are more senior high school students than junior middle school students. Conclusion: 1) Students in school have different degrees of mental health problems. 2) These mental health problems are different due to three different factors.
\end{abstract}

\section{Keywords}

Students, Mental Health Problems

\section{Introduction}

As we all know, mental health is as important as physical health. Mental health problems are different from physical diseases. They are a series of problems caused by people's inner spiritual factors, specifically, the central nervous system of the brain. Psychological research is divided into adult psychology and adolescent psychology. Previously, a psychological health survey was conducted on 1012 people from Liaoning Normal University, Anshan Normal University and Jilin Normal University. Results show that $89.43 \%$ of them had mild mental health problems [1]. At the same time, some people have done the mental health 
survey of middle school students in Yunnan, Guizhou, Sichuan, Shanxi, Chongqing and other five provinces with 1000 questionnaires. The results show that 10 to 30 percent of adolescents have varying levels of mental health problems [2]. Researchers have also done surveys on the mental health of elementary school students. The subjects of the survey were fourth-graders from Dazhou No. 1 Primary School in Sichuan province. The results showed that the number of students with different degrees of mental health problems also accounted for a certain proportion [3]. The purpose of this study is to focus on the mental health status of primary and middle school students in Hunan, Guangxi, Guangdong and Beijing. The difference between this survey and the previous ones is the location of the respondents: there were 77 in Hunan, 62 in Guangxi, 123 in Guangdong and 23 in Beijing. On the test papers, we set 10 polygraphs questions, with a total score of 20. Questionnaires with scores greater than or equal to 14 in that part are considered invalid. Only valid questionnaires were counted in this research.

\section{Objects and Methods}

\subsection{Survey Objects and Object Recruitment}

The objects are primary and middle school students (ages 6 to 19), with an average age of 14. The final total is 331 people. Among them, 159 are male and 172 are female. And there are 77 people in Hunan, 62 in Guangxi, 123 in Guangdong and 23 in Beijing. Primary school students (aged 6 to 11) average 7 years old, with a total number of 16 people. Junior high school students (15 to 12 years old) average 13 years old, with a total number of 121 people. High school students (16 to 19 years old) average 17 years old, with a total number of 176 people. During the period from 25th October 2018 to 10th December 2018, by scanning the QR code generated by the questionnaire star through WeChat or directly clicking the link generated after making the questionnaire on the questionnaire star, respondents can fill in the questionnaire randomly under the condition of anonymity. The survey focuses on students from first - and second-tier cities in China, including Hunan province, Guangxi province, Guangdong province and Beijing. When nearly 50 questionnaires are collected, the red envelope function is activated. Those who filled out the questionnaire with WeChat have a 20 percent chance of winning a red envelope of 5 yuan. When the number of people in the questionnaire reaches nearly 200, a high school student in Shenzhen is hired at a cost of RMB 400 to help forward the questionnaire to students in Shenzhen and ask them to fill it out.

\subsection{Measuring Tool}

Diagnostic mental health test (MHT). Zhou Bucheng, professor of east China normal university, translated, revised and published the "mental health diagnostic test (M H T) handbook" in 1991, based on Diagnostic Test of Restlessness scale compiled by Suzuki Qing, Japan. The scale is widely used in thousands of 
primary and secondary schools in more than 20 provinces and cities in China (except Hong Kong, Macao and Taiwan). Its scientificity, practicability and operability have been proved, and it has good reliability and validity. It has become a standardized mental health diagnostic test for Chinese students, and can be used to comprehensively detect the mental health status of primary and secondary school students, referred to as MHT. The scale contains eight dimensions of psychology: learning anxiety, anxiety about people, loneliness tendency, self-accusation tendency, allergy tendency, physical symptoms, terror tendency and impulse tendency. Each dimension scores 10, 0 to 4 is a low rating; 6 is rated medium; 8 to 10 is a high rating. The higher the score for each dimension, the worse the performance of that dimension. Also on the test papers, question 45 to 54 are polygraphs, with a total score of 20. Questionnaires with scores greater than or equal to 14 in that part are considered invalid. The initial MHT has 100 questions, one point per question. Through the revised questionnaire, each small question has 2 points, and each aspect has 5 questions. The ten questions on the validity test remain the same (lie detector test).

\subsection{Data Processing}

After the questionnaire was collected, all the original data were downloaded from the background of questionnaire star, and the Numbers software was used to make descriptive statistics and test for the data. In this survey, 6 invalid questionnaires with validity score of 14 were eliminated, and a total of 327 valid questionnaires were finally recovered. The IP address of the respondents was used to classify their region. The information of the research subjects was determined by setting blank-filling questions in the questionnaire to ask the gender, age and grade of the respondents.

\section{Results}

According to the data survey, the average score of the test subjects was 45.8 . The score ranges from 12 to 84 , with a minimum score of 12 and a maximum score of 84 . Variance is 227.13 .

\subsection{The Detection Rate of Mental Health Problems in School Students}

In this study, the detection rate of mental health problems refers to the ratio of the number of students with scores greater than or equal to 8 in any of the 8 dimensions of 331 students (see Table 1). As can be seen from Table 1, 34 of the 331 students in school have severe mental health problems, accounting for $10.27 \%$ of the total. Among them, 17 are male students, accounting for $10.69 \%$ of the total number of male students, and 17 are girls, accounting for $9.88 \%$ of the total number of girls. For study anxiety, male accounts for $41.50 \%$, and female accounts for $54.06 \%$. For anxiety about people, male accounts for $15.72 \%$, and female accounts for $19.18 \%$. For loneliness tendency, male accounts for 
$6.28 \%$ and female accounts for $7.55 \%$. For self-accusation tendency, male accounts for $28.93 \%$ and female accounts for $44.76 \%$. For allergy tendency, male accounts for $33.33 \%$ for male and female accounts for $48.83 \%$. For physical symptoms, male accounts for $30.18 \%$, and female accounts for $43.60 \%$. For terror tendency, male accounts for $5.66 \%$ and female accounts for $8.72 \%$. For impulsive tendency, male accounts for $10.69 \%$, and female accounts for $11.04 \%$.

\subsection{Regional Differences in Mental Health Problems among College Students (See Table 2)}

A total of 77 people were tested in Hunan, 62 in Guangxi, 123 in Guangdong and 23 in Beijing. The detection rate of mental health problems in a region refers to the proportion of the total number of students in a specific region who score more than or equal to 8 in any of the 8 dimensions of mental health for any student. Excluding other minority areas, the population of the psychological questionnaire survey is mainly concentrated in Hunan, Guangxi, Guangdong, Beijing and other four regions, with a total number of 285 people. Other regions (1 Macau, 3 Canada, 1 USA, 2 Korea, 4 Xinjiang, 1 Yunnan, etc.) have no reference value due to the small number of people, so we left it out (a total of 42 people). By comparing and analyzing the data of students' learning anxiety and allergy tendency, it is concluded that students in Beijing have a poor performance in the dimension of impulse tendency compared with students in the other three regions. Beijing students impulse tendency reaches $30.43 \%$, other areas are less than $10 \%$. Therefore, students who represent this area have more serious psychological problems of impulsive tendency. At the same time, students in Beijing performed better overall than those in the other three regions in terms of allergy tendency. The allergy tendency of students in Beijing was $17.36 \%$, and that of students in other areas was higher than $30 \%$.

\subsection{Mental Health Problems among Students of Different Ages (See Table 3)}

Because students of different ages may be at the same learning stage, so students aged 16 to 19 are classified as high school students in this survey. The average age of the high school test subjects is 17 . The variance between the ages is 0.68 . Students aged 12 to 15 are classified as junior middle school students. The average age of the junior middle school test subjects is 13 years old, and the variance between the ages is 1.25. Students aged 6 to 11 are classified as primary school students. The average age of the primary school test subjects is 7 years old, and the variance between the ages is 0.26 . According to the data, $31.25 \%$ of primary school students have learning anxiety, $48.76 \%$ of middle school students have learning anxiety, and $56.23 \%$ of high school students have learning anxiety. Therefore, the later the learning stage is, the greater the learning pressure of students will be. 
Table 1. Detection rate of mental health of students in school.

\begin{tabular}{cccc}
\hline & Male & Female & Total \\
\hline Total students number & 159 & 172 & 331 \\
No. of students on study anxiety & $66(41.50 \%)$ & $93(54.06 \%)$ & $159(48.03 \%)$ \\
No. of students on anxiety about people & $25(15.72 \%)$ & $33(19.18 \%)$ & $58(17.52 \%)$ \\
No. of students on loneliness tendency & $10(6.28 \%)$ & $13(7.55 \%)$ & $23(6.94 \%)$ \\
No. of students on self-accusation tendency & $46(28.93 \%)$ & $77(44.76 \%)$ & $123(37.16 \%)$ \\
No. of students on allergy tendency & $53(33.33 \%)$ & $84(48.83 \%)$ & $137(41.38 \%)$ \\
No. of students on physical symptoms & $48(30.18 \%)$ & $75(43.60 \%)$ & $123(37.16 \%)$ \\
No. of students on terror tendency & $9(5.66 \%)$ & $15(8.72 \%)$ & $24(7.25 \%)$ \\
No. of students on impulsive tendency & $17(10.69 \%)$ & $19(11.04 \%)$ & $36(10.87 \%)$
\end{tabular}

Table 2. Regional differences in mental health problems among school students (detection rate $[\mathrm{n}])$.

\begin{tabular}{ccccc}
\hline & Hunan & Guangxi & Guangdong & Beijing \\
\hline $\begin{array}{c}\text { Total students number } \\
\text { No. of students on study anxiety }\end{array}$ & $39(50.64 \%)$ & $32(51.61 \%)$ & $57(46.34 \%)$ & $13(56.52 \%)$ \\
No. of students on anxiety about & $17(22.07 \%)$ & $13(20.96 \%)$ & $19(15.44 \%)$ & $6(26.08 \%)$ \\
people & & & 123 & \\
$\begin{array}{c}\text { No. of students on loneliness tendency } \\
\text { No. of students on self-accusation }\end{array}$ & $5(6.49 \%)$ & $2(3.22 \%)$ & $9(7.31 \%)$ & $5(21.73 \%)$ \\
tendency & $22(28.57 \%)$ & $24(38.70 \%)$ & $52(42.27 \%)$ & $8(34.78 \%)$ \\
No. of students on allergy tendency & $46(59.74 \%)$ & $33(53.22 \%)$ & $44(35.77 \%)$ & $4(17.39 \%)$ \\
No. of students on physical symptoms & $9(11.68 \%)$ & $14(22.58 \%)$ & $5(4.06 \%)$ & $6(26.08 \%)$ \\
No. of students on terror tendency & $4(5.19 \%)$ & $4(6.45 \%)$ & $10(8.13 \%)$ & $4(17.39 \%)$ \\
No. of students on impulsive tendency & $7(9.09 \%)$ & $6(9.67 \%)$ & $14(11.38 \%)$ & $7(30.43 \%)$ \\
\hline
\end{tabular}

Table 3. Differences in learning stages of mental health problems among students (detection rate $[\mathrm{n}]$ ).

\begin{tabular}{|c|c|c|c|}
\hline & $\begin{array}{c}\text { Age } 6-11 \text {, } \\
\text { average } 7.56 \text {, } \\
\text { variance } 0.26\end{array}$ & $\begin{array}{c}\text { Age } 12-15, \\
\text { average } 13, \\
\text { variance } 1.25\end{array}$ & $\begin{array}{c}\text { Age } 16-19, \\
\text { average } 17.25 \text {, } \\
\text { variance } 0.68\end{array}$ \\
\hline Total students number & 16 & 121 & 176 \\
\hline No. of students on study anxiety & $5(31.25 \%)$ & $59(48.76 \%)$ & $90(51.13 \%)$ \\
\hline $\begin{array}{l}\text { No. of students on anxiety about } \\
\text { people }\end{array}$ & $5(31.25 \%)$ & $13(10.74 \%)$ & $36(20.45 \%)$ \\
\hline No. of students on loneliness tendency & $0(0 \%)$ & $9(7.43 \%)$ & $13(7.38 \%)$ \\
\hline $\begin{array}{l}\text { No. of students on self-accusation } \\
\text { tendency }\end{array}$ & $5(31.25 \%)$ & $45(37.19 \%)$ & $69(39.20 \%)$ \\
\hline No. of students on allergy tendency & $5(31.25 \%)$ & $47(38.84 \%)$ & $80(45.45 \%)$ \\
\hline No. of students on physical symptoms & $0(0 \%)$ & $7(5.78 \%)$ & $31(17.61 \%)$ \\
\hline No. of students on terror tendency & $0(0 \%)$ & $10(8.26 \%)$ & $12(6.81 \%)$ \\
\hline No. of students on impulsive tendency & $0(0 \%)$ & $14(11.57 \%)$ & $21(11.93 \%)$ \\
\hline
\end{tabular}




\section{Discussion}

\subsection{Gender Difference Leads to Different Mental Health Status}

The study found that $10.27 \%$ of the students have serious mental health problems. Problems focus on learning anxiety, self-accusation tendency, and physical symptoms. Among them, the number of boys with mental health problems is higher than that of girls. But boys are healthier overall than girls. The results are consistent with previous surveys [4]. Previous studies have suggested that this is because the hypothalamus is larger in men than it is in women. The hypothalamus is the part of the brain responsible for regulating emotions, sexual needs and questions adjustments. It controls our circadian cycles [4].

\subsection{Regional Differences Lead to Different Mental Health Conditions}

From the perspective of regional differences, the mental health problems of teenagers in second-tier cities (Hunan and Guangxi) are slightly more serious than those in first-tier cities (Guangdong and Beijing). According to the results of this study, this phenomenon can be specifically reflected in the fact that students in second-tier cities are under greater learning pressure than those in first-tier cities. The reason for this may be that household income in second-tier cities is lower than that in first-tier cities, which leads to that students in second-tier cities are under more study pressure than those in first-tier cities. According to others' survey, the family's financial income is related to the study pressure. The lower the family income is, the higher the student's academic pressure score [5]. Therefore, it is possible because that household income in first-tier cities is higher than that in second-tier cities, and students are under greater pressure in study.

\subsection{Age Difference Leads to Different Mental Health Status}

In terms of age difference, high school students are more serious than junior high school students and primary school students in all aspects, especially in learning anxiety. The reason for this phenomenon is that there are previous data showing that the study in high school is very intense. It can be seen from the aspect of mathematics that the difficulty of mathematics learning in high school has significantly improved for most students. This is not only due to the continuous improvement of the depth of inquiry of high school mathematics knowledge itself, but also greatly related to the surge in the number of related knowledge points [6]. Students who struggle to adjust face this anxiety. Learning anxiety affects people's anxiety in life.

\subsection{Specific Feasible Plan to Reduce the Negative Effect of Adolescents' Psychological Problems}

\subsubsection{Introspection-Regulates Allergic and Impulsive Tendencies}

Confucianism believes that students should positively control themselves, that's 
individual's subjective initiative. The psychological problems should be solved by controlling the events that lead to psychological conflicts or puzzles, or by the transformation of cognition and the positive cultivation of individuals. The Taoist way to solve the psychological problems is to give up the wisdom and keep the simplicity, to use and take nothing. The Buddhism solve the psychological problems by changing the survival puzzles into other aspects of problems. These methods have certain passivity. But if we can connect it with some psychological problems reasonably, it is an effective way to deal with them. Modern psychological research has proved that if people can reflect on themselves with correct moral norms, actively self-hint and prevent emotional over-excitement, they can effectively prevent various psychological problems caused by emotional runaway [7].

\subsubsection{The Moderation Way-Adjust All Aspects of Psychological Tendency}

A gentleman is master of his own inner world. He can adjust his own psychological experience of various external stimuli, and keep his inner world in balance at all times. Moderation, in modern terms, is properness. This is the attitude of a wise man towards life. It is of great help to adjust modern life style, regulate moral behavior and obtain success in life [7].

\subsubsection{Self-Cultivation-Regulating Anxiety}

"Internal sanctity" is to conduct moral cultivation and achieve the personality of sages. "Foreign king" is engaged in moral practice to make achievements. Strengthening moral cultivation, forming a correct outlook on life, world outlook and values, and developing a noble moral personality are the foundation of being a person, the conditions for one to make achievements, and one of the important signs of one's mental health. In the modern era when "instrumental rationality" is rampant, the value of modern people is lost, the interpersonal relationship is indifferent, the egoism is expanded, and the social ethos goes from bad to worse, thus resulting in the loss of people's spiritual home. In view of these disadvantages, we need to carry forward the essence of traditional culture to adjust and restrict people's mentality [7].

\subsubsection{Joy and Sorrow-Psychotherapy}

The method of dispelling sorrow and pleasing oneself is very valuable for reference. It is a kind of psychological therapy that helps people with mental illness to regulate their negative emotions through pleasing oneself. Wu Shiji in the Qing dynasty in the External treatment said "for the disease of the seven emotions, reading and listening to music are better than taking medicine." The method is to change the environment through the play and appreciation of landscape flowers and plants, as well as the love of literature and art, conversation, playing chess and painting, and the appropriate use of tea and wine, which are pleasing to the heart, so as to achieve the adjustment of depression, anxiety, tension and 
other psychological diseases [7].

\subsubsection{Limitations of the Study}

First, the prototype of MHT questionnaire is used in this survey. However, considering that most users will use WeChat to fill in the questionnaire, it is difficult for the respondents to take 25 - 30 minutes to complete the questionnaire. So, the ten questions for each dimension are reduced to five, and the lie-detector questions remained the same. Ensure that everyone can finish the test in about 10 minutes to prevent the test subjects have negative impact due to fatigue. Secondly, the questionnaire of this survey is forwarded on wechat moments in the form of questionnaire (snowball sampling). Therefore, the disadvantage of snowball sampling is that the survey object is limited to a group of people with similar idea attributes, which will cause serious underrepresentation. Because through each other's introduction, it tends to find a group of people with similar views. This group is often just a small circle of subgroups that the researchers want to study. What's more, the motivation of the interviewees often comes from human pressure, which may be the cause of the selective bias. Finally, the data results of this survey do not analyze the significance through statistics, so all the comparisons are directly judged by eyes without statistical analysis.

\section{Conclusion}

By investigating the mental health status of students in Hunan, Guangxi, Guangdong and Beijing, this survey found that girls are more serious than boys, second-tier cities are worse than first-tier cities and middle school students are more serious than primary school students. We can help students solve their mental health problems by pleasing themselves and eliminating depression.

\section{Conflicts of Interest}

The author declares no conflicts of interest regarding the publication of this paper.

\section{References}

[1] Zhao, Y. and Wang, J.Y. (2007) Investigation and Analysis of College Students' Mental Health Status in Normal Universities. Chinese Journal of Health Psychology, No. 1, 15.

[2] Yang, B., Xin, Y., Shi, Q. and Qi, J. (2000) Primary School Students Mental Health Survey Report. Journal of Da County Normal College, No. 4, 10.

[3] Li, H.Y. (2008) Survey and Analysis of Middle School Students' Mental Health. China Science and Education Innovation Guide, 231.

[4] Feinstein, S. (2012) Guess Who Has the Cleverest Brain-Men and Women Compete for Brain Power. In: To Explore the Mysteries of the Adolescent Brain-To Give Brain Science Research Methods of Adolescent Education, 56-57.

[5] Li, J.H., Feng, X.L., Mei, S.L. and Yao, D.L. (2007) An Investigation on the Influence of Learning Stress on the Mental Health of Junior Middle School Students. Medi- 
cine and Society, No. 2, 56-57.

[6] Shen, P. (2015) Use the Knowledge Classification Skillfully, Reduces the High School Mathematics Difficulty. Mathematics, Physics and Chemistry (Teaching and Learning) for Middle School Students, No. 7, 82.

[7] Zhang, Z.H. (2005) On the Value of Mental Health Education in Traditional Culture. Modern University Education, No. 5, 79-81. 